\title{
Black-Scholes Option Pricing Model
}

\author{
Nathan Coelen
}

June 6, 2002

\section{Introduction}

Finance is one of the most rapidly changing and fastest growing areas in the corporate business world. Because of this rapid change, modern financial instruments have become extremely complex. New mathematical models are essential to implement and price these new financial instruments. The world of corporate finance once managed by business students is now controlled by mathematicians and computer scientists.

In the early 1970's, Myron Scholes, Robert Merton, and Fisher Black made an important breakthrough in the pricing of complex financial instruments by developing what has become known as the Black-Scholes model. In 1997, the importance of their model was recognized world wide when Myron Scholes and Robert Merton received the Nobel Prize for Economics. Unfortunately, Fisher Black died in 1995, or he would have also received the award [Hull, 2000]. The Black-Scholes model displayed the importance that mathematics plays in the field of finance. It also led to the growth and success of the new field of mathematical finance or financial engineering.

In this paper, we will derive the Black-Scholes partial differential equation and ultimately solve the equation for a European call option. First, we will discuss basic financial terms, such as stock and option, and review the arbitrage pricing theory. We will then derive a model for the movement of a stock, which will include a random component, Brownian motion. Then, we will discuss some basic concepts of stochastic calculus that will be applied to our stock model. From this model, we will derive the Black-Scholes partial differential equation, and I will use boundary conditions for a European call option to solve the equation. 


\section{Definitions}

Financial assets are claims on some issuer, such as the federal government or a corporation, such as Microsoft. Financial assets also include real assets such as real estate, but we will be primarily concerned with common stock. Common stock represents an ownership in a corporation. Stocks provide a claim to the corporation's income and assets. A person who buys a financial asset in hopes that it will increase in value has taken a long position. A person who sells a stock before he/she owns it hoping that it decreases in value is said to be short an asset. People who take short positions borrow the asset from large financial institutions, sell the asset, and buy the asset back at a later time.

A derivative is a financial instrument whose value depends on the value of other basic assets, such as common stock. In recent years, derivatives have become increasingly complex and important in the world of finance. Many individuals and corporations use derivatives to hedge against risk. The derivative asset we will be most interested in is a European call option. A call option gives the owner the right to buy the underlying asset on a certain date for a certain price. The specified price is known as the exercise or strike price and will be denoted by $E$. The specified date is known as the expiration date or day until maturity. European options can be exercised only on the expiration date itself. Another common option is a put option, which gives the owner the right to sell the underlying asset on a certain date for a certain price.

For example, consider a July European call option contract on Microsoft with strike price $\$ 70$. When the contract expires in July, if the price of Microsoft stock is $\$ 72$ the owner will exercise the option and realize a profit of $\$ 2$. He will buy the stock for $\$ 70$ from the seller of the option and immediately sell the stock for $\$ 72$. On the other hand, if a share of Microsoft is worth $\$ 69$ the owner of the option will not exercise the option and it will expire worthless. In this case, the buyer would lose the purchase price of the option.

\section{Arbitrage}

One of the most fundamental theories to the world of finance is the arbitrage pricing theory. The theory states that two otherwise identical assets cannot sell at different prices. This also means that there are no opportunities to 
make an instantaneous risk-free profit. Here we are assuming the risk-free rate to be that of a bank account or a government bond, such as a Treasury bill.

To illustrate the concept of arbitrage, consider a simple example of a stock that is traded in the U.S. and in London. In the U.S. the price of the stock is $\$ 150$ and the asset sells for $£ 100$ in London, while the exchange rate is $\$ 1.60$ per pound. A person could make an instantaneous profit by simultaneously buying 100 shares of stock in New York and selling them in London. An instantaneous profit of

$$
100 *((\$ 1.60 * 100)-\$ 150)=\$ 1000
$$

is realized without risk.

\section{Hedging}

Three types of traders are attracted to derivative securities: speculators, arbitrageurs, and hedgers. Speculators take long or short positions in derivatives to increase their exposure to the market. They are betting that the underlying asset will go up or go down. Arbitrageurs find mispriced securities and instantaneously lock in a profit by adopting certain trading strategies like those discussed above. The last group is hedgers who take positions in derivative securities opposite those taken in the underlying asset in order to help manage risk. For example, consider an investor who owns 100 shares of Microsoft which is currently priced $\$ 62$. The person is worried that the stock might decline sharply in the next two months. The person could buy put options on Microsoft to sell 100 shares at a price of $\$ 60$. The person would pay the price of the options, but this would ensure that he could sell the stock for $\$ 60$ at expiration if the stock declines sharply. One very important hedging strategy is delta hedging. The delta, $\Delta$, of the option is defined as the change of the option price with respect to the change in the price of the underlying asset. In other words, delta is the first derivative of the option price with respect to the stock price:

$$
\Delta=\frac{\partial V}{\partial S}
$$

For example, suppose that the delta of a call option is .60 , the price of a stock is $\$ 100$ and the price of a call option is $\$ 10$. Imagine an investor who 
has sold 1 call option. The call option gives the buyer the right to buy 100 shares, since each option contract is for 100 shares. The seller's position could be hedged by buying $0.6 * 100=60$ shares. The gain (loss) on the option position would then tend to be offset by the loss (gain) on the stock position. If the stock price goes up by $\$ 1$ (producing a gain of $\$ 60$ on the shares purchased) the option price would tend to go up by $0.6 * \$ 1=\$ 0.6$ (producing a loss of $\$ 0.6 * 100=\$ 60$ on the call option written)[Hull, 2000].

\section{$5 \quad$ Stock Price Model}

Most people agree that stock prices move randomly because of the efficient market hypothesis. There are different forms of this hypothesis, but all say the same two things. First, the history of the stock is fully reflected in the present price. Second, markets respond immediately to new information about the stock. With the previous two assumptions, changes in a stock price follow a Markov process. A Markov process is a stochastic process where only the present value of the variable is relevant for predicting the future. So, our stock model states that our predictions for the future price of the stock should be unaffected by the price one week, one month, or one year ago.

As stated above, a Markov process is a stochastic process. In the real world, stock prices are restricted to discrete values, and changes in the stock price can only be realized during specified trading hours. Nevertheless, the continuous-variable, continuous-time model proves to more useful than a discrete model.

Another important observation is to note that the absolute change in the price of a stock is by itself, not a useful quality. For example, an increase of one dollar in a stock is much more significant on a stock worth $\$ 10$ than a stock worth $\$ 100$. The relative change of the price of a stock is information that is more valuable. The relative change will be defined as the change in the price divided by the original price.

Now consider the price of a stock $S$ at time $t$. Consider a small time interval $d t$ during which the the price of the underlying asset $S$ changes by an amount $d S$. The most common model separates the return on the asset, $d S / S$ into two parts. The first part is completely deterministic, and it is usually the risk free interest rate on a Treasury bill issued by the government. 
This part yields a contribution of

$$
\mu d t
$$

to $d S / S$. Here $\mu$ is a measure of the average rate of growth of the stock, also known as the drift. In this model $\mu$ is assumed to be the risk free interest rate on a bond, but it can also be represented as a function of $S$ and $t$. The second part of the model accounts for the random changes in the stock price due to external effects, such as unanticipated news. It is best modelled by a random sample drawn from a normal distribution with mean zero and contributes

$$
\sigma d B
$$

to $d S / S$. In this formula $\sigma$ is defined as the volatility of the stock, which measures the standard deviation of the returns. Like the term $\mu, \sigma$ can be represented as a function of $S$ and $t$. The $B$ in $d B$ denotes Brownian motion, which will be described in the next section. It is important to note that $\mu$ and $\sigma$ can be estimated for individual stocks using statistical analysis of historical prices. This is not of interest for our model. It is only important that $\mu$ and $\sigma$ are functions of $S$ and $t$. Putting this information together, we obtain the stochastic differential equation

$$
d S / S=\mu d t+\sigma d B
$$

Notice that if the volatility is zero the model implies

$$
d S / S=\mu d t
$$

When $\mu$ is constant this equation can be solved so that

$$
S=S_{0} e^{\mu t}
$$

where $S$ is the price of the stock at $t$ and $S_{0}$ is the price of the stock at $t=0$. This equation shows that when the variance of an asset is zero, the asset grows at a continuously compunded rate of $\mu$ per unit of time. 


\section{Brownian Motion}

The term that has not been discussed so far is the random term, $d B$. Brownian motion, which was originally used as a model for stock price movements in 1900 by L. Bachelier[Klebaner, 1998], is a stochastic process $B(t)$ characterized by the following three properties:

1. Normal Increments: $B(t)-B(s)$ has a normal distribution with mean 0 and variance $t-s$. Notice if $s=0$ that $B(t)-B(0)$ has normal distribution with mean 0 and variance $t$.

2. Independence of Increments: $B(t)-B(s)$ is independent of the past.

3. Continuity of Paths: $B(t), t>0$ are continuous functions of $t$.

These three properties alone define Brownian motion, but they also show why Brownian motion is used to model stock prices. Property 2 shows stock price changes will be independent of past price movements. This was an important assumption we made in our stock price model.

An occurence of Brownian motion from time 0 to $\mathrm{T}$ is called a path of the process on the interval $[0, \mathrm{~T}]$. There are five important properties of Brownian motion paths. The path $B(t), 0<t<T$

1. is a continuous function of $t$,

2. is not monotonic on any interval, no matter how small the interval is,

3. is not differentiable at any point,

4. has infinite variation on any interval no matter how small it is,

5. has quadratic variation on $[0, \mathrm{t}]$ equal to $t$, for any $t$.

Together properties 1 and 3 state that although Brownian motion paths are continuous, the $\Delta B(t)$ over interval $\Delta t$ is much larger than $\Delta t$ as $\Delta t \rightarrow$ 0 . Properties 4 and 5 show the distinction between functions of Brownian motion and normal, smooth functions. The variation of a function over the interval $[\mathrm{a}, \mathrm{b}]$ is defined as

$$
V_{g}([a, b])=\sup \sum_{i=1}^{n} \mid g\left(t_{i}\right)-g\left(t_{i-1}\right)
$$


where the supremum is taken over partitions:

$$
a=t_{0}<t_{1}<\ldots<t_{n}=b .
$$

It can be seen that smooth functions are of finite variation while Brownian motion is of infinite variation. Quadratic variation plays a very important role with Brownian motion and stochastic calculus. Quadratic variation is defined for a function $g$ over the interval $[0, t]$ as

$$
[g, g](t)=\lim \sum_{i=1}^{n}\left(g\left(t_{i}\right)-g\left(t_{i-1}\right)\right)^{2},
$$

where the limit is taken over partitions:

$$
0=t_{0}<t_{1}<\ldots<t_{n}=t
$$

Quadratic variation plays no role in standard calculus due to the fact that continuous functions of finite variation have quadratic variation of 0.

\section{Stochastic Calculus}

In this section I will introduce stochastic integrals with respect to Brownian motion. These stochastic integrals are commonly called Itô integrals. In order to procede with the derivation of the Black-Scholes formula we need to define thestochastic integral $\int_{0}^{T} X(t) d B(t)$. If $X(t)$ is a constant, $c$, then $\int_{0}^{T} c d B(t)=c(B(T)-B(0))$. The integral over $(0, T]$ should be the sum of integrals over subintervals $\left[0, a_{1}\right),\left(a_{1}, a_{2}\right),\left(a_{2}, a_{3}\right), \ldots,\left(a_{n-1}, T\right]$. So if $X(t)$ takes values $c_{i}$ on each subinterval then the integral of $X$ with respect to $B$ is easily defined.

First we consider the integrals of simple processes $e(t)$ which depend on $t$ and not on $B(t)$. A simple deterministic process $e(t)$ is a process for which there exist times $0=t_{0}<t_{1}<t_{2}<\ldots<t_{n}=T$ and constants $c_{0}, c_{1}, c_{2}, c_{n-1}$, such that

$$
e(t)=\left\{\begin{array}{cc}
c_{0} & \text { if } t=0 \\
c_{i} & \text { if } t_{i}<t \leq t_{i+1}, i=0, \ldots, n-1 .
\end{array}\right.
$$

Therefore, the Itô integral $\int_{0}^{T} X(t) d B(t)$ is defined as the sum

$$
\int_{0}^{T} e(t) d B(t)=\sum_{i=0}^{n-1} c_{i}\left(B\left(t_{i+1}\right)-B\left(t_{i}\right)\right) .
$$


The Itô integral of simple processes is a random variable with the following four properties.

1. Linearity. If $X(t)$ and $Y(t)$ are simple processes and $\alpha$ and $\beta$ are constants then

$$
\int_{0}^{T}(\alpha X(t)+\beta Y(t)) d B(t)=\alpha \int_{0}^{T} X(t) d B(t)+\beta \int_{0}^{T} Y(t) d B(t) .
$$

2. The integral of the indicator function of an interval $I_{[a, b]}(t)=1$ when $t \in[a, b]$, and zero otherwise is just $B(b)-B(a), 0<a<b<T$,

$$
\int_{0}^{T} I_{[a, b]}(t) d B(t)=B(b)-B(a)
$$

3. Zero mean property. $E \int_{0}^{T} X(t) d B(t)=0$.

4. Isometry Property.

$$
E\left(\int_{0}^{T} X(t) d B(t)\right)^{2}=\int_{0}^{T} E\left(X^{2}(t)\right) d t .
$$

The definition of the Itô integral can be extended to processes $X(t)$ that can be approximated by sequences $e^{n}$ of simple processes in the sense that

$$
E\left(\int_{0}^{T}\left|e^{n}(t)-X(t)\right|^{2} d t\right) \rightarrow 0
$$

as $n \rightarrow \infty$. In that case, we define

$$
\int_{0}^{T} X(t) d B=\lim _{n \rightarrow \infty} \int_{0}^{T} e^{n} t d B .
$$

This is a sound definition beecause the limit does not depend on the approximating sequence. Also the integral that arises this way still satisfies properties 1-4 above.

Now that we have defined the Itô integral of simple processes we wish to define Itô integral of other processes. It can be shown that if a general predictable process satifies certain conditions, the eneral process is a limit in probability of siple predictable processes we discussed earlier. The Itô integral of general predictable processes is defined as a limit of integrals of 
simple processes. If $X(t)$ is a predictable process such that $\int_{0}^{T} X^{2}(t) d t<\infty$ then the Itô integral $\int_{0}^{T} X(t) d B(t)$ is defined and satisfies the above four properties.

For example, we find the $\int_{0}^{T} B(t) d B(t)$. We let $0=t_{0}<t_{1}<t_{2}<\ldots<$ $t_{n}=T$ be a partition of $[0, T]$ then

$$
e^{n}(t)=\sum_{i=0}^{n-1} B\left(t_{i}\right) I_{\left[t_{i}, t_{i+1}\right]}(t)
$$

Then for any $n, e^{n}(t)$ is a simple process. We can take a sequence of the partitions such that $\max _{i}\left(t_{i+1}-t_{i}\right) \rightarrow 0$ as $n \rightarrow \infty$. The Itô integral of this simple function is given by

$$
\int_{0}^{T} e^{n}(t) d B(t)=\sum_{i=0}^{n-1} B\left(t_{i}\right)\left(B\left(t_{i+1}\right)-B\left(t_{i}\right)\right)
$$

We now show that this sequence of integrals converges in probability and identify the limit. Adding and subtracting $B^{2}\left(t_{i+1}\right)$, we obtain

$$
B\left(t_{i}\right)\left(B\left(t_{i+1}\right)-B\left(t_{i}\right)\right)=\frac{1}{2}\left(B^{2}\left(t_{i+1}\right)-B^{2}\left(t_{i}\right)-\left(B\left(t_{i+1}\right)-B\left(t_{i}\right)\right)^{2}\right) .
$$

and

$$
\begin{aligned}
\int_{0}^{T} e^{n}(t) d B(t) & =\frac{1}{2} \sum_{i=0}^{n-1}\left(B^{2}\left(t_{i+1}\right)-B^{2}\left(t_{i}\right)\right)-\frac{1}{2} \sum_{i=0}^{n-1}\left(B\left(t_{i+1}\right)-B\left(t_{i}\right)\right)^{2}(5) \\
& =\frac{1}{2} B^{2}(T)-\frac{1}{2} B^{2}(0)-\frac{1}{2} \sum_{i=0}^{n-1}\left(B\left(t_{i+1}\right)-B\left(t_{i}\right)\right)^{2}
\end{aligned}
$$

since the first sum is a telescpopic sum. Notice that from property 5 of Brownian motion patht the second sum converges to the limit $T$. Therefore, $\int_{0}^{T} e^{n}(t) d B(t)$ converges, and the limit is

$$
\int_{0}^{T} B(t) d B(t)=\lim _{n \rightarrow \infty} \int_{0}^{T} e^{n}(t) d B(t)=\frac{1}{2} B^{2}(T)-\frac{1}{2} T .
$$

This example illustrates the difference between deterministic calculus and stochastic calculus. The quadratic variation of continuous functions, $x(t)$, of finite variation we work with in standard calculus is 0 . Therefore, if we were calculating the integral of $\int_{0}^{T} x(t) d x(t)$ the same way as above the term 
$\sum_{i=0}^{n-1}\left(x\left(t_{i+1}\right)-x\left(t_{i}\right)\right)^{2}$ would converge to the limit 0 . So the $\int_{0}^{T} x(t) d x(t)$ simply equals $\frac{1}{2} x^{2}(T)$.

Now we need to discuss one of the main tools of stochastic calculus, Itô's formula, which is the stochastic counterpart of the chain rule in Calculus. Recall that Brownian motion has quadratic variation on $[0, \mathrm{t}]$ equal to $t$, for any $t$. This can also be expressed as the following

$$
\int_{0}^{t}(d B(s))^{2}=\int_{0}^{t} d s=t \text { or in differential notation }(d B(t))^{2}=d t .
$$

Using this property and applying Taylor's formula, Itô's formula states that if $f(x)$ is twice differentiable function then for any $t$

$$
f(B(t))=f(0)+\int_{0}^{t} f^{\prime}(B(s)) d B(s)+\frac{1}{2} \int_{0}^{t} f^{\prime \prime}(B(s)) d s .
$$

Note that Itô's formula in differential notation becomes

$$
d\left(f(B(t))=f^{\prime} B(t)\right) d B(t)+\frac{1}{2} f^{\prime \prime}(B(t)) d t .
$$

Next we define an Itô process. Let $Y(t)$ be an Itô integral process

$$
Y(t)=\int_{0}^{t} X(s) d B(s) .
$$

An Itô process is an Itô integral plus an adapted continuous process of finite variation. Process $Y$ is called an Itô process if for any $0 \leq t \leq T$ it can be represented as

$$
Y(t)=Y(0)+\int_{0}^{t} \mu(s) d s+\int_{0}^{t} \sigma(s) d B s .
$$

More generally, if $Y$ is an Itô process represented above then it has a stochastic differential on $[o, T]$

$$
d Y(t)=\mu(t) d t+\sigma(t) d B(t)
$$

for $0 \leq t \leq T$. The function $\mu$ is often called the drift coefficient and the function $\sigma$ is called the diffusion coefficient.

One last very important case for us to consider is for functions of the form $f(X(t), t)$. If $f(x, t)$ is a twice continuously differentiable in $\mathrm{x}$, and 
continuously differentiable in t and $X(t)$ represnets an Itô process, then

$$
\begin{aligned}
d f(x(t), t)=\frac{\partial f}{\partial x}( & X(t), t) d X(t)+\frac{\partial f}{\partial t}(X(t), t) d t \\
& +\frac{1}{2} \sigma^{2}(X(t), t) \frac{\partial^{2} f}{\partial x^{2}}(X(t), t) d t .
\end{aligned}
$$

We will use this case of Itô's formula to compute the Black-Scholes partial differential equation in the next section.

\section{Derivation of the Equation}

In the next two sections, the price of a derivative security, $V(S, t)$ is derived. The model for a stock we derived in section 5 satisfies a Itô process defined in equation 6 . Therefore, we let the function $V(S, t)$ be twice differentiable in $S$ and differentiable in $t$. Applying equation 7 from above we have

$$
d V(S, t)=\frac{\partial V}{\partial S} d S+\frac{\partial V}{\partial t} d t+\frac{1}{2} \sigma^{2} S^{2} \frac{\partial^{2} V}{\partial S^{2}} d t
$$

Plugging into equation 8 for $d S$ with Equation 1 we have

$$
d V(S, t)=\frac{\partial V}{\partial S}(\mu S d t+\sigma S d B)+\frac{\partial V}{\partial t} d t+\frac{1}{2} \sigma^{2} S^{2} \frac{\partial^{2} V}{\partial S^{2}} d t .
$$

This simplifies to

$$
d V(S, t)=\sigma S d B \frac{\partial V}{\partial S}+\left(\mu S \frac{\partial V}{\partial S}+\frac{\partial V}{\partial t}+\frac{1}{2} \sigma^{2} S^{2} \frac{\partial^{2} V}{\partial S^{2}}\right) d t
$$

Now set up a portfolio long one option, $V$, and short an amount $\frac{\partial V}{\partial S}$ stock. Note from above that this portfolio is hedged. The value of this portfolio, $\pi$, is

$$
\pi=V-\frac{\partial V}{\partial S} S
$$

The change, $d \pi$, in the value of this portfolio over a small time interval $d t$ is given by

$$
d \pi=d V-\frac{\partial V}{\partial S} d S
$$


Now plugging equations 9 and 1 into equation 11 for $d V$ and $d S$ we get

$$
\begin{array}{r}
d \pi=\sigma S d B \frac{\partial V}{\partial S} d B+\left(\mu S \frac{\partial V}{\partial S}+\frac{\partial V}{\partial t}+\frac{1}{2} \sigma^{2} S^{2} \frac{\partial^{2} V}{\partial S^{2}}\right) d t \\
-\frac{\partial V}{\partial S}(\mu S d t+\sigma S d B)
\end{array}
$$

This simplifies to

$$
d \pi=\left(\frac{\partial V}{\partial t}+\frac{1}{2} \sigma^{2} S^{2} \frac{\partial^{2} V}{\partial S^{2}}\right) d t
$$

It is important to note that this portfolio is completely riskless because it does not contain the random Brownian motion term. Since this portfolio contains no risk it must earn the same as other short-term risk-free securities. If it earned more than this, arbitrageurs could make a profit by shorting the riskfree securities and using the proceeds to buy this portfolio. If the portfolio earned less arbitrageurs could make a riskless profit by shorting the portfolio and buying the risk-free securities. It follows for a riskless portfolio that

$$
d \pi=r \pi d t
$$

where $\mathrm{r}$ is the risk free interst rate. Substituting for $d \pi$ and $\pi$ from equations 13 and 10 yields

$$
\left(\frac{\partial V}{\partial t}+\frac{1}{2} \sigma^{2} S^{2} \frac{\partial^{2} V}{\partial S^{2}}\right) d t=r\left(V-S \frac{\partial V}{\partial S}\right) d t .
$$

Further simplification yields the Black-Scholes differential equation

$$
\frac{\partial V}{\partial t}+\frac{1}{2} \sigma^{2} S^{2} \frac{\partial^{2} V}{\partial S^{2}}+r S \frac{\partial \partial S}{-r V}=0
$$

\section{Solution for a European Call}

In order to solve the Black-Scholes equation derived in the last section we need to consider final and boundary conditions, or else the partial differential equation does not have a unique solution. For this project we will concern ourselves with a European call, $C(S, t)$ with exercise price $E$ and expiry date $T$. 
The final condition at time $t=T$ can be derived from the definition of a call option. If at expiration $S>E$ the call option will be worth $S-E$ because the buyer of the option can buy the stock for $E$ and immediately sell it for $S$. If at expiration $S<E$ the option will not be exercised and it will expire worthless. At $t=T$, the value of the option is known for certain to be the payoff

$$
C(S, T)=\max (S-E, 0) .
$$

This is the final condition for our differential equation.

In order to find boundary conditions we consider the value of $C$ when $S=0$ and as $S \rightarrow \infty$. If $S=0$ then it is easy to see from equation 1 that $d S=0$, and therefore, $S$ will never change. If at expiry $S=0$ then from equation 17 the payoff must be 0 . Consequently, when $S=0$ we have

$$
C(0, T)=0 .
$$

Now when $S \rightarrow \infty$ it becomes more and more likely the option will be exercised and the payoff will be $S-E$. The exercise price becomes less and less important as $S \rightarrow \infty$, so the value of the option is equivalent to

$$
C(S, T) \approx S \text { as } S \rightarrow \infty .
$$

In order to solve the Black-Scholes equation we need to transform the equation into an equation we can work with. The first step is to get rid of the $S$ and $S^{2}$ terms in equation 16. In order to do this consider the change of variables

$$
\begin{gathered}
S=E e^{x} \\
t=T-\frac{\tau}{\frac{1}{2} \sigma^{2}} \\
V=E v(x, \tau) .
\end{gathered}
$$

Using the Chain rule from Calculus for transforming partial derivatives for functions of two variables we have

$$
\frac{\partial V}{\partial S}=\frac{\partial V}{\partial x} \frac{\partial x}{\partial S}+\frac{\partial V}{\partial \tau} \frac{\partial \tau}{\partial S}
$$




$$
\frac{\partial V}{\partial t}=\frac{\partial V}{\partial x} \frac{\partial x}{\partial t}+\frac{\partial V}{\partial \tau} \frac{\partial \tau}{\partial t}
$$

Looking at equations 20, 21, and 22 it can be shown that

$$
\frac{\partial \tau}{\partial t}=-\frac{1}{2} \sigma^{2} \quad \frac{\partial x}{\partial t}=0 \quad \frac{\partial x}{\partial S}=\frac{1}{S} \quad \frac{\partial \tau}{\partial S}=0
$$

Plugging these into equation 23 and equation 24 yields

$$
\begin{gathered}
\frac{\partial V}{\partial S}=\frac{E}{S} \frac{\partial V}{\partial x} \\
\frac{\partial V}{\partial t}=-\frac{1}{2} \sigma^{2} E \\
\frac{\partial^{2} V}{\partial S^{2}}=\frac{E}{S^{2}} \frac{\partial^{2} v}{\partial x^{2}}-\frac{E}{S^{2}} \frac{\partial V}{\partial x} .
\end{gathered}
$$

Substituting equation 25, equation 26, and equation 27 into the BlackScholes partial differential equation gives the differential equation

$$
\frac{\partial V}{\partial \tau}=\frac{\partial^{2} v}{\partial x^{2}}+(k-1) \frac{\partial V}{\partial x}-k v
$$

where

$$
k=\frac{r}{\frac{1}{2} \sigma^{2}}
$$

The initial condition $C(S, T)=\max (S-E, 0)$ is transformed into

$$
v(x, 0)=\max \left(e^{x}-1,0\right) .
$$

Now we apply another change of variable and let

$$
v=e^{\alpha x+\beta \tau} u(x, \tau) .
$$

Then by simple differntiation we have

$$
\frac{\partial V}{\partial \tau}=\beta e^{\alpha x+\beta \tau} u+e^{\alpha x+\beta \tau} \frac{\partial u}{\partial \tau}
$$




$$
\begin{gathered}
\frac{\partial V}{\partial x}=\alpha e^{\alpha x+\beta \tau} u+e^{\alpha x+\beta \tau} \frac{\partial u}{\partial x} \\
\frac{\partial^{2} v}{\partial x^{2}}=\alpha\left(\alpha e^{\alpha x+\beta \tau} u+e^{\alpha x+\beta \tau} \frac{\partial u}{\partial x}\right)+\alpha e^{\alpha x+\beta \tau} \frac{\partial u}{\partial x}+e^{\alpha x+\beta \tau} \frac{\partial^{2} u}{\partial x^{2}} .
\end{gathered}
$$

Substituting these partials into equation 28 yields

$$
\beta u+\frac{\partial u}{\partial \tau}=\alpha^{2} u+2 \alpha \frac{\partial u}{\partial x}+\frac{\partial^{2} u}{\partial x^{2}}+(k-1)\left(\alpha u+\frac{\partial u}{\partial x}\right)-k u .
$$

We can get rid of the of the $u$ terms and the $\frac{\partial u}{\partial x}$ terms by carefully choosing values of $\alpha$ and $\beta$ such that

$$
\beta=\alpha^{2}+(k-1) \alpha-k
$$

and

$$
2 \alpha+k-1=0 \text {. }
$$

We can rearrange these equations so they can be written

$$
\alpha=-\frac{1}{2}(k-1) \text { and } \beta=-\frac{1}{4}(k+1)^{2} .
$$

We now have the tranformation from $v$ to $u$ is

$$
v=e^{-\frac{1}{2}(k-1) x-\frac{1}{4}(k+1)^{2} \tau} u(x, \tau)
$$

resulting in the simple diffusion equation

$$
\frac{d u}{d \tau}=\frac{d^{2} u}{d x^{2}} \text { for }-\infty<x<\infty, \tau>0 .
$$

Our initial condition has now been changed as well to

$$
u_{0}(x)=u(x, 0)=\max \left(e^{\frac{1}{2}(k+1) x}-e^{\frac{1}{2}(k-1) x}, 0\right) .
$$

The solution to the simple diffusion equation obtained above is well-known to be

$$
u(x, \tau)=\frac{1}{\sqrt{2 \pi}} \int_{-\infty}^{\infty} u_{0}(s) e^{-\frac{(x-s)^{2}}{4 \tau}} d s
$$


where $u_{0}(x, 0)$ is given by equation 31 . In order to solve this integral it is very convenient to make a change of variable

$$
y=\frac{s-x}{\sqrt{2 \tau}}
$$

so that

$$
u(x, \tau)=\frac{1}{\sqrt{2 \pi}} \int_{-\infty}^{\infty} u_{0}(y \sqrt{2 \tau}+x) e^{-\frac{y^{2}}{2}} d y
$$

Substituting our intitial condition into this equation results in

$$
\begin{aligned}
u(x, \tau)= & \frac{1}{\sqrt{2 \pi}} \int_{-\frac{x}{\sqrt{2 \tau}}}^{\infty} e^{\frac{1}{2}(k+1)(y \sqrt{2 \tau}+x)} e^{-\frac{y^{2}}{2}} d y \\
& -\frac{1}{\sqrt{2 \pi}} \int_{-\frac{x}{\sqrt{2 \tau}}}^{\infty} e^{\frac{1}{2}(k-1)(y \sqrt{2 \tau}+x)} e^{-\frac{y^{2}}{2}} d y .
\end{aligned}
$$

In order to solve this we will solve each integral separately. The first integral can be solved by completing the square in the exponent. The exponent of the first integral is

$$
-\frac{1}{2} y^{2}+\frac{1}{2}(k+1)(x+y \sqrt{2 \pi}) .
$$

Factoring out the $-\frac{1}{2}$ gives us

$$
-\frac{1}{2}\left(y^{2}-[k+1] y \sqrt{2 \tau}-[k+1] x\right) .
$$

Separating out the term that is not a function of $y$, and adding and subtracting terms to set up a perfect square yields

$$
\frac{1}{2}(k+1) x-\frac{1}{2}\left(y^{2}-[k+1] y \sqrt{2 \tau}+\left(\frac{[k+1] \sqrt{2 \tau}}{2}\right)^{2}-\left(\frac{[k+1] \sqrt{2 \tau}}{2}\right)^{2}\right]
$$

which can be written

$$
\frac{1}{2}(k+1) x-\frac{1}{2}\left(y-\frac{[k+1] \sqrt{2 \tau}}{2}\right)^{2}+\frac{1}{2}\left(\frac{[k+1] \sqrt{2 \tau}}{2}\right)^{2}
$$


and simplified to

$$
\frac{1}{2}(k+1) x-\frac{1}{2}\left(y-\frac{[k+1] \sqrt{2 \tau}}{2}\right)^{2}+\frac{(k+1)^{2} \tau}{4} .
$$

Thus the first integral reduces to

$$
I_{1}=\frac{e^{\frac{1}{2}(k+1) x}}{\sqrt{2 \pi}} \int_{-\frac{x}{\sqrt{2 \tau}}}^{\infty} e^{\frac{1}{4}(k+1)^{2} \tau} e^{-\frac{1}{2}\left(y-\frac{1}{2}[k+1] \sqrt{2 \tau}\right)^{2}} d y .
$$

Now substituting

$$
z=y-\frac{1}{2}[k+1] \sqrt{2 \tau}
$$

results in

$$
\begin{aligned}
I_{1} & =\frac{e^{\frac{1}{2}(k+1) x}+\frac{1}{4}(k+1)^{2} \tau}{\sqrt{2 \pi}} \int_{-\frac{x}{\sqrt{2 \tau}}-\frac{1}{2}(k+1) \sqrt{2 \tau}}^{\infty} e^{-\frac{1}{2} z^{2}} d z \\
& =\frac{e^{\frac{1}{2}(k+1) x}+\frac{1}{4}(k+1)^{2} \tau}{\sqrt{2 \pi}} N(d 1)
\end{aligned}
$$

where

$$
d_{1}=\frac{x}{\sqrt{2 \tau}}+\frac{1}{2}(k+1) \sqrt{2 \tau}
$$

and

$$
N(x)=\frac{1}{\sqrt{2 \pi}} \int_{-\infty}^{x} e^{-\frac{1}{2} y^{2}} d y
$$

is the cumulative distribution function for the normal distribution.

The calculation of the second integral $I_{2}$ is identical to that of $I_{1}$, except that $(\mathrm{k}-1)$ replaces $(\mathrm{k}+1)$ throughout. Finally, we work our way backwards with

$$
v(x, \tau)=e^{-\frac{1}{2}(k-1) x-\frac{1}{4}(k+1)^{2} \tau} u(x, \tau)
$$

and then substituting the inverse transformations

$$
x=\log \left(\frac{S}{E}\right)
$$




$$
\begin{gathered}
\tau=\frac{1}{2} \sigma^{2}(T-t) \\
C=E v(x, \tau)
\end{gathered}
$$

we finally obtain the desired result

$$
C(S, t)=S N\left(d_{1}\right)-E e^{-r(T-t)} N\left(d_{2}\right)
$$

where

$$
d_{1}=\frac{\log \left(\frac{S}{E}\right)+\left(r+\frac{1}{2} \sigma^{2}\right)(T-t)}{\sigma \sqrt{T-t}}
$$

and

$$
d_{2}=\frac{\log \left(\frac{S}{E}\right)+\left(r-\frac{1}{2} \sigma^{2}\right)(T-t)}{\sigma \sqrt{T-t}} .
$$

\section{Conclusion}

The important point to note about the derivation of the Black-Scholes differential equation is that we never specified a specific type of derivative security we were trying to find the price for until we set up boundary conditions for a European call. This means that a person can use the Black-Scholes differential equation to solve for the price of any type of option only by changing the boundary conditions.

The Black-Scholes model truly revolutionized the world of finance. For the first time the model has given traders, hedgers, and investors a standard way to value options. The model also has also caused a huge growth in the importance of financial engineering in the world of finance. Today, mathematicians are building models to maximize portfolio returns while minimizing risk. They are also building sophisticated computer programs to search for inefficiencies in the market. The world of finance is becoming built on mathematics and the Black-Scholes model was the beginning of this mathematical revolution. 


\section{Bibliography}

[Baxter, 1996] Baxter, Martin and Andrew Rennie. Financial Calculus: An Introduction to Derivative Pricing. Cambridge, England: Cambridge University Press, 1996.

[Hull, 2000] Hull, John C. Options, Futures, and Other Derivatives. Upper

Saddle River, New Jersey: Prentice Hall, 2000.

[Klebaner, 1998] Klebaner, Fima C. Introduction to Stochastic Calculus with Applications. London:Imperial College Press, 1998.

[Ross, 1999] Ross, Sheldon. An Introduction to Mathematical Finance. Cambridge, England: Cambridge University Press, 1999.

[Wilmott, 1995] Wilmott, Paul, Sam Howison, and Jeff Dewynne. The Mathematics of Financial Derivatives. Cambridge, England: Cambridge University Press, 1995. 